\title{
Estilo interpersonal controlador del entrenador, frustración de las necesidades psicológicas básicas, y burnout en futbolistas infantiles
}

\author{
Controlling Coach Interpersonal Style, Basic Psychological \\ Need Thwarting, and Burnout in Young Soccer Players
}

\author{
Isabel Castillo, Lorena González, Priscila Fabra, Juan Mercé e Isabel Balaguer
}

Universidad de Valencia

\begin{abstract}
Resumen: En el presente trabajo, basado en la Teoría de las Necesidades Psicológicas Básicas (BPNT; Deci y Ryan, 2000), se puso a prueba un modelo con la siguiente secuencia: Estilo interpersonal controlador del entrenador P Frustración de las necesidades psicológicas básicas P Burnout. Participaron 725 futbolistas varones de la categoría infantil con edades comprendidas entre los 11 y los 13 años $(M=12.6 ; D T=.54)$ que completaron los instrumentos que evaluaban las variables de interés. Los resultados del análisis de ecuaciones estructurales informaron que la percepción del estilo controlador del entrenador actuaba de predictor positivo de la frustración de las tres necesidades y que cada una de estas tres últimas, se asociaba positivamente con el burnout.

Palabras clave: estilo controlador del entrenador, frustración de las necesidades psicológicas básicas, burnout, futbolistas infantiles.
\end{abstract}

Abstract: Based on the basic psychological needs theory (BPNT; Deci \& Ryan, 2000), in the present study a model with the following sequence was tested: controlling interpersonal coaching style $\mathrm{P}$ basic psychological need thwarting P burnout. Participants were 725 young male soccer players from category under 14 years old (Infantil), aged between 11 and 13 ( $M=$ 12.6; $S D=.54)$ that completed the questionnaires tapping the variables of interest. Results of structural equation analyses informed that perception of a controlling interpersonal coaching style was a positive predictor of need thwarting for competence, autonomy and relatedness; thwarting for these three needs were positively associated with burnout.

Key words: controlling coaching style, basic psychological need thwarting, burnout, young soccer players.

\section{Introducción}

La Teoría de las Necesidades Psicológicas Básicas (BPNT; Deci y Ryan, 2000), una de las mini-teorías de la Teoría de la Autodeterminación (SDT; Deci y Ryan, 2000) defiende que las necesidades de competencia (sensación de dominio/maestría mediante la interacción eficaz con el entorno), autonomía (sensación de que uno es el origen o la fuente de la propia acción) y relación (sensación de estar conectado a los demás, sentir afecto hacia y desde otros) juegan un importante papel en el desarrollo del bienestar psicológico y del funcionamiento óptimo (Deci y Ryan, 2000). También postula que los contextos sociales pueden facilitar este desarrollo o dificultarlo, a través de la satisfacción o frustración de estas necesidades. En concreto, en aquellos contextos en los que se apoya la autonomía se favorece la satisfacción de las necesidades psicológicas (sensaciones que se producen cuando se percibe que se tienen cubiertas las necesidades psicológicas) y el bienestar; mientras que en los entornos controladores se promueve la frustración de las necesidades (sensaciones que se producen cuando las personas perciben que sus necesidades psicológicas son mermadas por otros) y el malestar (Bartholomew, Ntoumanis, Ryan, Bosch y ThøgersenNtoumani, 2011; Ryan y Deci, 2000).

Dirección de correspondencia: Isabel Castillo - Facultad de Psicología Departamento de Psicología Social- Avda. Blasco Ibañez, 21. 46010 Valencia (España)- Tlf: 9638645 77-Email: Isabel.Castillo@uv.es
Adaptándolo al contexto deportivo, la SDT sugiere que cuando los entrenadores tienen un estilo interpersonal de apoyo a la autonomía (el entrenador apoya activamente las iniciativas de los deportistas y crea condiciones para que experimenten un sentido de volición, elección y desarrollo personal) se favorecerá la satisfacción de las necesidades y el bienestar psicológico, mientras que cuando establezcan un estilo controlador (el entrenador actúa de manera coercitiva y autoritaria para imponer a los deportistas su forma de pensar y actuar) se facilitará la frustración de las necesidades y se propiciará el malestar (Bartholomew, Ntoumanis y Thøgersen-Ntoumani, 2010).

Hasta la fecha, la investigación en el contexto deportivo se ha centrado predominantemente en estudiar las relaciones entre la percepción del estilo interpersonal de apoyo a la autonomía del entrenador sobre la satisfacción de las necesidades psicológicas básicas y de éstas últimas sobre el bienestar o el malestar de los deportistas; obteniéndose apoyo general para los postulados de la BPNT (Balaguer, 2007; 2010). Sin embargo, poco se conoce en la actualidad sobre las consecuencias negativas del estilo interpersonal controlador sobre el malestar, y menos todavía sobre las relaciones secuenciales postuladas por la BPNT en esta ruta en la que se dificulta el funcionamiento psicológico: estilo interpersonal controlador del entrenador $\mathrm{P}$ la frustración de cada una de las necesidades 
psicológicas básicas $\mathrm{P}$ malestar (véase Balaguer et al., en prensa; Bartholomew, Ntoumanis, Ryan, Bosch et al., 2011). De ahí que nuestro objetivo en el presente trabajo se centre en el estudio de esta secuencia tomando como indicador del malestar el burnout, constructo que ha sido considerado como un síndrome psicosocial que se caracteriza por la presencia de agotamiento emocional y físico, devaluación deportiva y disminución del sentido de logro en la actividad en cuestión (Raedeke y Smith, 2004).

En las pocas investigaciones realizadas hasta la fecha en las que se han estudiado las relaciones entre la percepción del estilo controlador del entrenador, la frustración de las necesidades psicológicas y el burnout, tanto a nivel transversal (Bartholomew, Ntoumanis, Ryan, Bosch et al., 2011), como longitudinal (Balaguer et al., en prensa) la frustración de las necesidades se ha evaluado a nivel global y los resultados han ofrecido apoyo general a los efectos negativos de este estilo interpersonal del entrenador sobre el funcionamiento psicológico.

En el presente trabajo, a diferencia de los dos anteriores, se estudiaron a nivel independiente las relaciones de la frustración de cada una de las necesidades psicológicas básicas con los antecedentes y consecuentes de la BPNT. En concreto se puso a prueba un modelo con la siguiente secuencia: Estilo interpersonal controlador del entrenador P Frustración de cada una de las necesidades psicológicas básicas P Burnout. Se hipotetizó que el estilo interpersonal controlador del entrenador actuaría como predictor positivo de la frustración de cada una de las necesidades (competencia, autonomía y relación) y que cada una de estas ultimas variables se asociaría positivamente con el burnout.

\section{Método}

\section{Participantes}

La muestra estuvo compuesta por 725 futbolistas varones de la categoría infantil pertenecientes a 42 equipos de fútbol base de la provincia de Valencia, con edades comprendidas entre los 11 y los 13 ańos $(M=12.6 ; D T=.54)$ que entrenaban una media de 2.26 días a la semana $(D T=1.63)$.

\section{Instrumentos}

La percepción del estilo interpersonal controlador del entrenador se evaluó con la versión española (Castillo et al., 2010) de la escala de Conductas Controladoras del Entrenador (CCBS, Bartholomew et al., 2010), compuesta por 15 ítems divididos en cuatro subescalas (uso controlador de recompensas, atención condicional negativa, intimidación, excesivo control personal). Cada ítem se inicia con la frase: "En mi equipo de fútbol" y las respuestas se recogen en una escala tipo Likert que oscila desde totalmente en desacuerdo (1), a totalmente de acuerdo (7). Para el presente estudio se utilizó la puntuación media en la escala total.

La frustración de las necesidades psicológicas básicas se evaluó mediante la versión española (Balaguer et al., 2010) de la Escala de Frustración de las Necesidades Psicológicas (PNTS; Bartholomew, Ntoumanis, Ryan y Thøgersen-Ntoumani, 2011). La escala tiene 12 ítems agrupados en tres subescalas que evalúan el grado en el que los deportistas perciben que se frustran sus necesidades de competencia, de autonomía y de relación. Cada ítem se inicia de la siguiente forma: "En mi equipo de fútbol...". Las respuestas se recogen en una escala tipo Likert de 7 puntos con un rango que oscila desde totalmente en desacuerdo (1) a totalmente de acuerdo (7).

La percepción de burnout se evaluó mediante la versión española (Balaguer et al., 2011) del Cuestionario de Burnout Deportivo (ABQ; Raedeke y Smith, 2001). Los 15 ítems del cuestionario se dividen en tres subescalas de cinco ítems cada una (cansancio físico y emocional, devaluación del deporte, y disminución del sentido del logro). Las respuestas se recogen en una escala tipo Likert que oscila desde casi nunca (1) a casi siempre (5). En este estudio se utilizó la puntuación media en la escala total.

Investigaciones previas han confirmado una adecuada fiabilidad de los instrumentos utilizados: CCBS (Bartholomew et al., 2010; Castillo et al., 2010), PNTS (Balaguer et al., 2010; Bartholomew, Ntoumanis, Ryan y Thøgersen-Ntoumani, 2011) y $A B Q$ (Balaguer et al., 2011; Bartholomew, Ntoumanis, Ryan y Thøgersen-Ntoumani, 2011; Raedeke y Smith, 2001).

\section{Procedimiento}

Los jugadores cumplimentaron los instrumentos transcurrido un mes y medio desde el inicio de la temporada con el objeto de tener tiempo suficiente para una adecuada percepción del estilo interpersonal del entrenador y durante una sesión de entrenamiento para evitar los efectos potenciales de la competición en las respuestas. Todos los jugadores firmaron el consentimiento para participar en el estudio. Durante la recogida de la información, al menos un investigador estuvo presente y en todo momento se respetó el anonimato de los participantes.

\section{Resultados}

\section{Descriptivos y Fiabilidad de las Escalas}

Los descriptivos y los coeficientes de fiabilidad (alfa de Cronbach) de las medidas del estudio se presentan en la Tabla 1. Todos los coeficientes de fiabilidad cumplen el criterio mínimo de .70 determinado para las escalas del dominio psicológico (Nunnally, 1978), oscilando el rango entre .70 y .88. 
Tabla 1. Descriptivos y consistencia interna de las variables del estudio

\begin{tabular}{lcccc}
\hline Variable & Rango & Media & $D T$ & $\Delta$ \\
\hline Estilo Controlador & $1-7$ & 2.43 & .92 & .80 \\
Frustración Competencia & $1-7$ & 2.65 & 1.33 & .75 \\
Frustración Autonomía & $1-7$ & 3.23 & 1.31 & .70 \\
Frustración Relación & $1-7$ & 2.56 & 1.36 & .73 \\
Burnout & $1-5$ & 1.93 & .71 & .88 \\
\hline
\end{tabular}

\section{Modelo de Ecuaciones Estructurales}

El modelo estructural hipotetizado se ha puesto a prueba utilizando el método de máxima verosimilitud del LISREL 8.54 (Jöreskog y Sörbom, 2003). Los resultados mostraron un adecuado ajuste de los datos: $\mathbb{\nabla}^{2}(161)=370.14, p<.01$; CFI = 97; $\mathrm{NNFI}=.96 ; \mathrm{RMSEA}=.063$. Los parámetros de la solución estandarizada se presentan en la Figura 1.

Los resultados informaron que la percepción del estilo controlador del entrenador actuaba de predictor positivo de la frustración de las necesidades de competencia, de autonomía y de relación; y que estas tres últimas a su vez se asociaban positivamente con el burnout. El modelo explicó un 34\% de la varianza del burnout.

Figura 1. Solución estandarizada del modelo estructural hipotetizado de la asociación entre el estilo controlador, la frustración de las necesidades psicológicas básicas y el burnout. Nota. Todos los coeficientes son significativos $(p<.01)$.

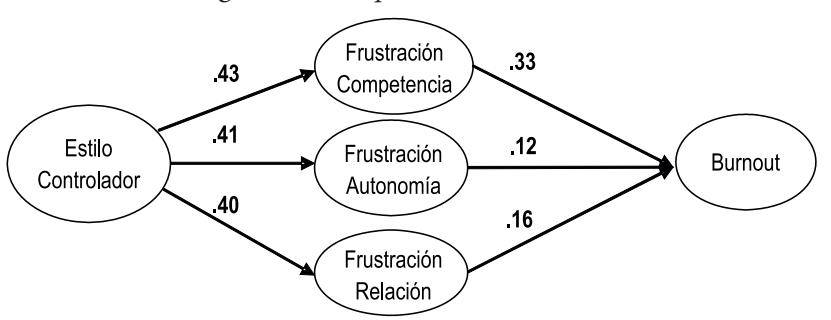

\section{Conclusiones y Discusión}

En el presente estudio se puso a prueba un modelo a la base de los postulados de la BPNT (Deci y Ryan, 2000) en el que se analizó el poder predictivo de la percepción del estilo controlador del entrenador sobre la frustración de cada una de las necesidades psicológicas básicas, y el de estas últimas sobre un indicador de malestar (burnout).

En la misma línea que en estudios previos, transversales (Bartholomew, Ntoumanis, Ryan, Bosch et al., 2011) y longitudinales (Balaguer et al., en prensa), en los que se operacionalizó la frustración de las necesidades a nivel global, en este trabajo se ha visto que el estilo controlador tiene un poder predictivo significativo sobre la frustración de cada una de las necesidades psicológicas básicas y que cada una de éstas a su vez actuó como predictor positivo del burnout.

En conclusión, en este trabajo se muestra que la creación de estilos interpersonales controladores por parte de los entrenadores tiene implicaciones negativas sobre las experiencias psicológicas de los jóvenes futbolistas y enfatiza la importancia de formar a los entrenadores en la creación de entornos positivos (http://www.projectpapa.co.uk/).

\section{Referencias}

Costa, P. T., y McCrae, R. R. (1992). Revised NEO Personality Inventory (NEO-PI-R) and NEO Five-Factor Inventory (NEO-FFI) professional manual. Odessa, FL: Psychological Assessment Resources.

Balaguer, I. (2007). Clima motivacional, calidad de la implicación y bienestar psicológico: Una propuesta de intervención en equipos deportivos. En A. Blanco y J. Rodríguez Marín (Eds.), Intervención Psicosocial (pp. 136-162). Madrid: Prentice-Hall.

Balaguer, I. (2010). Clima motivacional y bienestar. Actuación de los entrenadores. En J.M. Buceta y E. Larumbe (Eds.), Experiencias en Psicología del Deporte (pp. 181-202). Madrid: Dykinson.

Balaguer, I., Castillo, I., Duda, J.L., Quested, E., \& Morales, V. (2011). Predictores socio-contextuales y motivacionales de la intención de continuar participando: Un análisis desde la SDT en danza. Revista Internacional de Ciencias del Deporte, VII, 25, 305-319.

Balaguer, I., Castillo, I., Mercé, J., Ródenas, L.T., Rodríguez, A., García-
Merita, M., \& Ntoumanis, N. (2010). Escala de Frustración de las Necesidades Psicológicas, en el contexto deportivo: Análisis de las propiedades psicométricas. VII Congreso Iberoamericano de Psicología. Oviedo, Asturias. 20-24 julio.

Balaguer, I., González, L., Fabra, P., Castillo, I., Mercé, J., \& Duda, J.L. (en prensa). Coaches' interactive style, psychological needs, and the emotional welfare of young footballers: A longitudinal analysis. Journal of Sports Sciences.

Bartholomew, K. J., Ntoumanis, N., \& Thøgersen-Ntoumani, C. (2010). The controlling interpersonal style in a coaching context: Development and initial validation of a psychometric scale. Journal of Sport \& Exercise Psychology, 32, 193-216.

Bartholomew, K. J., Ntoumanis, N., Ryan, R. M., \& Thøgersen-Ntoumani, C. (2011). Psychological need thwarting in the sport context: Assessing the darker side of athletic experience. Journal of Sport \& Exercise Psy- 
chology, 33, 75-102.

Bartholomew, K. J., Ntoumanis, N., Ryan, R., Bosch, J., \& ThogersenNtoumani, C. (2011). Self-determination theory and diminished human functioning: The role of interpersonal control and psychological need thwarting. Personality and Social Psychology Bulletin, 37, 14591473.

Castillo, I., Fabra, P., Marcos, D., González, L., Bartholomew, K.J., Fuentes, A., \& Balaguer, I. (2010). El Estilo Controlador del Entrenador: Análisis de las propiedades psicométricas. Simposio sobre "Adaptación y Validación de Cuestionarios en Psicología del Deporte". VII Congreso Iberoamericano de Psicología. Oviedo, Asturias. 20-24 julio.

Deci, E.L., \& Ryan, R.M. (2000). The "what" and "why" of goal pursuits: Human needs and the self-determination of behaviour. Psychological Inquiry, 11, 227-268.
Jöreskog, K.G., \& Sörbom, D. (2006), LISREL 8.80 [Computer software]. Chicago: Scientific Software International.

Nunnaly, J.C. (1978). Psychometric theory (2nd ed.). Nueva York: McGrawHill.

Raedeke, T.D., \& Smith, A. L. (2001). Development and preliminary validation of a measure of athlete burnout. Journal of Sport \& Exercise Psychology, 23, 281-306.

Raedeke, T.D., \& Smith, A.L. (2004). Coping resources and athlete burnout: an examination of stress mediated and moderation hypotheses. Journal of Sport \& Exercise Psychology, 26, 525-541.

Ryan, R.M., \& Deci, E.L. (2000). The darker and brighter sides of human existence: Basic psychological needs as a unifying concept. Psychological Inquiry, 11, 319-338. 